\title{
2'-5'-Oligoadenylate synthetase 1 polymorphisms are associated with tuberculosis: a case-control study
}

\author{
Shouquan Wu' ${ }^{1}$, Yu Wang ${ }^{1}$, Guo Chen ${ }^{1,2}$, Miaomiao Zhang ${ }^{1}$, Minggui Wang ${ }^{1}$ and Jian-Qing He ${ }^{1 *}$
}

\begin{abstract}
Background: 2'-5'-Oligoadenylate synthetase 1 (OAS1) plays an important role in inflammatory immune reactions. OAS1 polymorphisms have been associated with increased susceptibility to various diseases. We investigated the association of polymorphisms in OAS1 with tuberculosis (TB).

Methods: A total of 1215 TB cases and 1114 healthy controls were enrolled from two independent studies. Genotyping was conducted using the improved multiplex ligase detection reaction (iMLDR) method. Associations between OAS1 polymorphisms (rs2240190, rs1131454, 10,774,671 and 11,066,453) and TB risk were established based on distributions of allelic frequencies using different genetic models.

Results: Significant association was observed between rs10774671, rs1131454 and TB. In the initial study, the G allele of rs 10774671 was a significantly protective factor against TB $(P=0.006)$ and the genotype of $G G$ differed significantly between TB patients and controls under the codominant model $(P=0.008)$ after Bonferroni correction. In the validation study, we also observed that the rs $10774671 \mathrm{G}$ allele $(P=0.001)$ and $\mathrm{GG}$ genotype $(P=0$. 001) were associated with TB. In addition, we found that the rs1131454 $\mathrm{G}$ allele $(P=0.004)$ and $\mathrm{GG}$ genotype $(P=0.001)$ were protective against TB in the Chinese Han population.
\end{abstract}

Conclusions: We report novel associations of polymorphisms in OAS1 with TB in the Chinese Tibetan and Han populations. Similar studies in different populations and functional studies are warranted to confirm our results.

Keywords: Alleles, Tuberculosis, 2'-5'-Oligoadenylate synthetase 1, Polymorphism, Association study

\section{Introduction}

Tuberculosis (TB) is an infectious disease that constitutes a major global health problem. It is a major cause of morbidity and mortality globally, particularly in Asia and Africa, and ranks alongside human immunodeficiency virus (HIV) as a leading cause of death worldwide [1]. In 2017, an estimated 1.7 billion individuals were newly infected with the causative agent of TB [1]. However, only $5-15 \%$ of them will develop TB during their lifetime [2]. The outcome of TB infection is affected by many factors, such as malnutrition, co-infection with other pathogens, exposure to microbes and previous vaccination [3]. It was reported that host genetic factors

\footnotetext{
* Correspondence: jianqhe@gmail.com

'Department of Respiratory and Critical Care Medicine, West China Hospital, Sichuan University, No. 37, Guo Xue Alley, Chengdu 610041, Sichuan,

People's Republic of China

Full list of author information is available at the end of the article
}

play a crucial role in determining an individual's susceptibility to TB [4]. Recently, a number of genes potentially associated with TB susceptibility have been analysed in case-control studies.

Studies performed in mouse models and in patients indicate an important role of interferon (IFN) during TB infection [5, 6]. IFN- $\alpha$, IFN- $\beta$ and IFN- $\gamma$ play significant immunomodulatory roles in the protection of the host against infections. As the main mediator of the type II immune response, IFN- $\gamma$ is critical for controlling infections by intracellular pathogens, such as Mycobacteria [7]. Specifically, IFN- $\gamma$ plays a vital role in resistance to Mycobacterium tuberculosis (MTB) by activating macrophages, monocytes and Th1 cells [8]. 2'-5'-oligoadenylate synthetases (OASs) are IFN-inducible enzymes that play vital roles in the innate immune response against viruses. The OAS family includes OAS1, OAS2, OAS3, 
and OAS-like (OASL) protein. The OAS1 gene encodes OAS1, which is an extensively characterized enzyme induced by IFNs [9]. Binding of IFNs to their specific receptors results in induction of $O A S 1$ gene expression [10]. OAS1 is activated by the presence of doublestranded RNA and stimulates the oligomerisation of ATP into $2^{\prime}, 5^{\prime}$-linked oligoadenylates (2-5A) [11]. 2-5A can bind to latent ribonuclease L (RNase L), which then dimerizes into the active form. This active form facilitates apoptosis [12], attenuates proliferation [13] and inhibits protein synthesis [13]. It is possible that polymorphisms in OAS1 influence the expression of IFN- $\gamma$, the elimination of $M T B$, and ultimately affect the development of TB [14, 15].

Other OAS family members including OAS2, OAS3, and OASL also have been the focus of much research. OAS2 encodes the p69 and p71 IFN-induced isoforms [16]. OAS2 play an important role in the control of bacterial infection through direct interaction with patternrecognition receptors. It also has immunomodulatory function through immune cell receptor interaction [16]. In terms of the ability to synthesize 2-5A, OAS3 shows higher activity than OAS1 [17]. OAS3 is specialized for binding long dsRNA. The major function of OAS3 during infection is to produce 2-5A activators of RNaseL [18]. In contrast to other OAS family members, OASL lacks any synthetic activity. OASL has the ability to regulate type I IFN responses when infected with pathogens. It has been suggested that OASL could act as a regulator in the control of antiviral innate immunity through IFN signalling [19].

Single-nucleotide polymorphisms (SNPs) can regulate $O A S 1$ function at multiple levels, including expression, alternative splicing and enzyme activity. So far, among the OAS gene cluster, OAS1 polymorphisms have been the most studied and were reported to influence susceptibility to various diseases. For example, SNPs in OAS1 have been identified as candidates for susceptibility to viral infections, such as West Nile virus [20], hepatitis C [21], Chikungunya [22], dengue [23] and measles [24]. Previous data also indicated that OAS1 polymorphisms and haplotypes were potential risk factors for autoimmune conditions, including type 1 diabetes [25] and multiple sclerosis [26]. In addition, $O A S 1$ polymorphisms were reported to be associated with respiratory infection [27]. However, no previous studies have examined the association between $O A S 1$ and TB.

Collectively, these observations prompted us to propose the hypothesis that $O A S 1$ polymorphisms confer susceptibility to TB. The purpose of the study was to evaluate the prevalence of polymorphisms within the OAS1 gene in TB cases and healthy controls from the Chinese Tibetan and Han populations.

\section{Methods}

\section{Population study}

The initial study comprised 613 patients with TB and 602 healthy controls recruited from the People's Hospital of the Aba Tibetan Autonomous Prefecture. In the independent validation study, we selected 571 TB cases and 543 healthy controls based on the same inclusion and exclusion criteria from the Chinese Han population. All subjects were unrelated ethnic Chinese.

The patients' selection criteria were: 1) written consent and agreement to participate the study; 2) having a diagnosis of TB; 3 ) age $\geq 18$ years. The diagnosis of TB was based on sputum smear tests, sputum culture, clinical symptoms and radiological and histological pathologic examinations. Patients with HIV, hepatitis B or C, diabetes, immune-mediated disorders or other lung diseases were excluded from our study. Controls were individuals without history of $\mathrm{TB}$ and active $\mathrm{TB}$ infection that had undergone routine medical examination.

Informed consents were obtained from all individual participants included in the study. The study was approved by the ethical committee of the West China Hospital of Sichuan University.

\section{SNP selection and genotyping}

Tag SNPs in the study were downloaded from the Chinese Han in Beijing database of HapMap (http:// hapmap.ncbi.nlm.nih.gov/index.html.en, HapMap Data Rel 27 Phase II + III, on NCBI B36 assembly, dbSNP b126) in a region stretching from 1000 base pairs upstream and 1000 base pairs downstream of OAS1; other SNPs of OAS1 were also selected from previous studies [27, 28]. Tag SNPs were filtered according to the following criteria: (i) Hardy-Weinberg equilibrium (HWE) test $P \geq 0.05$; (ii) $\mathrm{r}^{2}$ of pairwise linkage disequilibrium (LD) $\leq 0.8$ and (iii) minor allele frequency $(\mathrm{MAF}) \geq 0.05$.

A sample of $5 \mathrm{ml}$ of peripheral blood was obtained from each participant and placed in a test tube containing EDTA. We then isolated genomic DNA from the collected whole blood samples using a genomic DNA purification kit, in accordance with the manufacturer's instructions (Axygen Scientific Inc., Union City, CA, USA). Genotyping was conducted using PCR and the improved multiple ligase detection reaction (iMLDR). Five percent of samples were re-genotyped to verify the initial results.

\section{Statistical analysis}

HWE and the differences in sex between the case and control were evaluated by the Chi-squared test. Unpaired $t$-tests were used to evaluate the significance of 
differences in age between the case and control groups. Genotype and allele frequencies of SNPs in the TB group vs. those in the control group were calculated by logistic regression. As the genetic determinants of TB were ageand gender-dependent [29, 30], logistic regression was used to adjust for gender and age. Odds ratio (OR) and 95\% confidence interval ( $95 \% \mathrm{CI}$ ) were examined to estimate the magnitude of the risk. LD and haplotype analyses were conducted using the SHEsis online software platform (http://analysis.bio-x.cn). A 2-sided $P$ value of less than 0.05 was considered statistically significant and $P$ values were conservatively Bonferroni-corrected for multiple comparisons. Power calculation was performed $(\alpha=0.05)$ by Quanto software (version 1.2.4, May 2009). The power of the two cohorts to detect relative risks (RR) of 2.0, 3.0 and 4.0 using the four SNPs studied under an allele genetic model were estimated by the Quanto software and are listed in Table S1. The two cohorts have reasonable power $(>80 \%)$ to detect genetic factors with RR 2.0 and above under the allele model in all four SNPs (Table S1). Gene-gene interaction was detected using the multifactor dimensionality reduction (MDR) software and MDR permutation testing module (http://www.epistasis.org and http://sourceforge.net/projects/mdr/files/mdrpt). We performed an eQTL analysis by using the GTEx Portal database (https://gtexportal.org/home/). All analyses were conducted using the Statistical Package for the Social Sciences (SPSS; SPSS Inc., Chicago, IL, USA), version 19.0.

Genotype data of rs10774671 in the Han Chinese in Beijing (CHB) were obtained from the HapMap project and whole-genome Expression data were obtained from the Gene Expression Omnibus of PubMed. rs10774671 genotype data were downloaded from the 1000 Genomes Browser (https://www.ncbi.nlm.nih.gov/variation/tools/1000genomes/). DNA samples were collected from participants living in the residential community at Beijing Normal University. As a result, a total of 40 individuals provided genotypic data of rs10774671. We then used the mRNA data obtained from lymphoblastoid cell lines (extracted from Gene Expression Omnibus of Pubmed, accession number GSE6536) to analyze the associations between rs10774671 and OAS1 mRNA expression levels [31].

\section{Results}

Demographic characteristics of the study groups

In the initial study, the number of cases was 613 $($ male $=392$; female $=221)$ and the controls was 602 $($ male $=392 ;$ female $=210)$. The validation cohort comprised 584 cases $($ male $=299$; female $=285)$ and 543 controls $($ male $=266$; female $=277)$. The mean age was $34.53 \pm 14.54$ years for the case group and $34.63 \pm 13.85$ years for the control group in the initial study. There were no significant differences between the case and control groups regarding age and sex $(P>0.05)$ in this cohort. However, the mean age was significantly different between cases (mean age $=$ $36.50 \pm 15.49)$ and controls (mean age $=39.35 \pm 15.13$ ) in the validation cohort $(P=0.002)$.

\section{Analysis of HWE and association of OAS1 SNPs with TB}

A total of four SNPs (rs2240190, rs1131454, 10,774,671 and $11,066,453)$ were selected for analysis. Two of these four were tag SNPs (rs1131454 and rs10774671). All four SNPs did not deviate from HWE. The MAFs of SNPs were more than 0.05 . Table 1 provides basic information on the four SNPs in our study.

The allele frequencies in the two groups are shown in Table 2. The genotype frequencies of the OAS1 polymorphisms under different genetic models are summarized in Table 3. In the initial cohort, a significant association was only detected in the allele frequency of rs10774671 and the $\mathrm{G}$ allele acted as a protective factor against $\mathrm{TB}$ $(P=0.006, \quad$ OR $=0.74,95 \%$ CI: $0.59-0.92)$. Compared with the AA genotype, the frequency of the GG genotype of rs10774671 polymorphism in the case group significantly differed from the controls under the codominant model (GG vs. AA: $P=0.008, \mathrm{OR}=0.41$, 95\% CI: 0.21-0.80), suggesting that rs10774671 decreased the risk of TB.

To verify the results of the Tibetan population, we analyzed an independent population of Chinese Han individuals. We found that rs10774671 was also significantly associated with $\mathrm{TB}$ in the Chinese Han population. Compared with allele A, the $\mathrm{G}$ allele was more common in the control group $(P=0.001, \mathrm{OR}=0.72,95 \% \mathrm{CI}$ : $0.59-0.87)$. We also found that the GG genotype was a protective factor against TB under codominant (GG VS.

Table 1 Characteristics of the 4 SNPs in OAS1 in the present study

\begin{tabular}{|c|c|c|c|c|c|c|}
\hline \multirow[t]{2}{*}{ SNP } & \multirow[t]{2}{*}{ Position } & \multirow[t]{2}{*}{ Region } & \multicolumn{2}{|c|}{ Tibetan population } & \multicolumn{2}{|c|}{ Han population } \\
\hline & & & MAF & HWE & MAF & HWE \\
\hline rs2240190 (C > A) & $113,346,127$ & Intron 1 & 0.190 & 0.975 & 0.190 & 0.783 \\
\hline $\operatorname{rs} 1131454(A>G)$ & $113,348,870$ & Exon 3 & 0.406 & 0.973 & 0.379 & 0.924 \\
\hline rs10774671 (A > G) & $113,357,193$ & Exon 7 splice acceptor site & 0.237 & 0.294 & 0.210 & 0.819 \\
\hline rs11066453 (A > G) & $113,365,621$ & Intron 7 & 0.136 & 0.807 & 0.132 & 0.848 \\
\hline
\end{tabular}

Abbreviation: SNP single nucleotide polymorphism, MAF minor allele frequency, HWE Hardy Weinberg equilibrium 
Table 2 Allele distribution of OAS1 polymorphisms in the two populations

\begin{tabular}{|c|c|c|c|c|c|c|c|c|}
\hline \multirow[t]{2}{*}{ SNP } & \multicolumn{4}{|c|}{ Tibetan population } & \multicolumn{4}{|c|}{ Han population } \\
\hline & Cases, n (\%) & Controls, n (\%) & $P^{\#}$ & $\mathrm{OR}^{\#}(95 \% \mathrm{Cl})$ & Cases, n (\%) & Controls, n (\%) & $P^{\#}$ & $\mathrm{OR}^{\#}(95 \% \mathrm{Cl})$ \\
\hline \multicolumn{9}{|c|}{$\mathrm{rs} 2240190(\mathrm{C}>\mathrm{A})$} \\
\hline$C$ & $941(76.8)$ & $954(79.2)$ & & & 918(81.0) & $864(80.0)$ & & \\
\hline A & $285(23.2)$ & $250(20.8)$ & 0.128 & $1.16(0.96-1.41)$ & 216(19.0) & $216(20.0)$ & 0.556 & $0.94(0.76-1.16)$ \\
\hline \multicolumn{9}{|c|}{$\operatorname{rs} 1131454(A>G)$} \\
\hline A & $778(63.5)$ & $726(60.3)$ & & & $704(62.1)$ & $610(56.5)$ & & \\
\hline G & $448(36.5)$ & 478(39.7) & 0.110 & $0.88(0.74-1.03)$ & $430(37.9)$ & $470(43.5)$ & $0.004^{*}$ & $0.78(0.66-0.93)$ \\
\hline \multicolumn{9}{|c|}{ rs10774671(A > G) } \\
\hline A & $1051(85.7)$ & 984(81.6) & & & 896(79.0) & 793(73.4) & & \\
\hline G & $175(14.3)$ & $222(18.4)$ & $0.006^{*}$ & $0.74(0.59-0.92)$ & $238(21.0)$ & $287(26.6)$ & $0.001^{*}$ & $0.72(0.59-0.87)$ \\
\hline \multicolumn{9}{|c|}{ rs11066453 (A > G) } \\
\hline A & $1171(95.5)$ & 1124(93.4) & & & $984(86.8)$ & $937(86.8)$ & & \\
\hline$G$ & $55(4.5)$ & $80(6.6)$ & 0.022 & $0.66(0.47-0.94)$ & $150(13.2)$ & $143(13.2)$ & 0.807 & $1.03(0.81-1.32)$ \\
\hline
\end{tabular}

SNP single nucleotide polymorphism, $\mathrm{Cl}$ confidence interval, $\mathrm{OR}$ odds ratio

\#adjusted by sex and age

*Bonferroni correction was performed with $p \leq 0.0125(0.05 / 4)$ considered significant

AA: $P=0.001, \mathrm{OR}=0.40,95 \% \mathrm{CI}: 0.23-0.69)$ and recessive (GG VS. AA+GA: $P=0.005, \mathrm{OR}=0.46,95 \% \mathrm{CI}$ : 0.27-0.79) models (Table 4). In addition, rs1131454 was associated with TB susceptibility in the validation study. Compared with the control group, the TB group had lower proportions of the $\mathrm{G}$ allele $(P=0.004, \mathrm{OR}=0.78$, 95\% CI: 0.66-0.93) and GG genotype (codominant model: GG vs. AA, $P=0.001$, OR $=0.52$, 95\% CI: $0.36-$ 0.76 ; recessive model: $\mathrm{GG}$ vs. $\mathrm{AA}+\mathrm{GA}, P=0.002, \mathrm{OR}=$ 0.59, 95\% CI: 0.42-0.82).

\section{Linkage disequilibrium and haplotype analyses}

Information on LD for these four SNPs in the Chinese Tibetan population is shown in Fig. 1. LD among these four SNPs in the OAS1 gene was evaluated by the LD test using $\mathrm{r}^{2}$, which confirmed that these SNPs were not in $\operatorname{LD}\left(\mathrm{r}^{2}<0.5\right)$. No haplotypes were associated with TB in the two studies (Table 4).

\section{Gene-gene interactions}

rs2240190, rs1131454 and rs10774671 formed the best gene-gene interaction model with a testing balanced accuracy of $57.61 \%$ and cross-validation consistency of 10 / 10. It was confirmed statistically significant using 1000 -fold permutation testing $(P=0.001)$.

\section{eQTL and gene expression analysis for rs10774671}

eQTL analysis using the GTEx Portal database showed significant eQTL effects of rs10774671 $(P<0.001)$ on OAS1 expression in whole blood from 369 individuals. The $40 \mathrm{CHB}$ were divided into three groups according to rs10774671genotypic data. In brief, $15 \mathrm{CHB}$ had the AA genotype, 20 had the GA genotype and 5 had the
GG genotype. As shown in Fig. 2, GEO2R analysis revealed that the rs10774671 polymorphism was associated with OAS1 expression levels after adjustment for multiple comparisons $(P<0.001)$.

\section{Discussion}

In China, the ethnic Tibetan population is more likely to be infected with TB than the Han population [32, 33]. It is possible that host genetic difference is one of the bases of TB susceptibility. In this study, four SNPs in OAS1 (rs2240190, rs1131454, 10,774,671 and 11,066,453) were investigated in Chinese Han and Chinese Tibetan patients with TB. Our data showed, for the first time, that the G allele and the GG genotype of rs10774671 in OAS1 were associated with TB in the two populations.

OAS1 is an enzyme that plays an important role in innate antiviral defence [34]. This enzyme is activated by the presence of double-stranded or single-stranded RNA with a secondary structure and catalyses the oligomerisation of ATP into 2-5A [35]. These processes activate latent RNase $\mathrm{L}$, which degrades viral and cellular RNA and blocks protein synthesis. In accordance with this function, human OAS1 mainly affects susceptibility to viral infections $[35,36]$. Given the importance of the OAS1 protein, SNPs in the OAS1 gene may affect susceptibility to infectious diseases. In the present study, we demonstrated that polymorphisms in OAS1 were associated with TB. It is unclear why the heightened antiviral enzyme activity in individuals was associated with $\mathrm{TB}$, but this association may be explained as follows. One explanation is that the major role of the OAS proteins is as immune regulators in innate immunity, although previous studies indicated that they also play important 


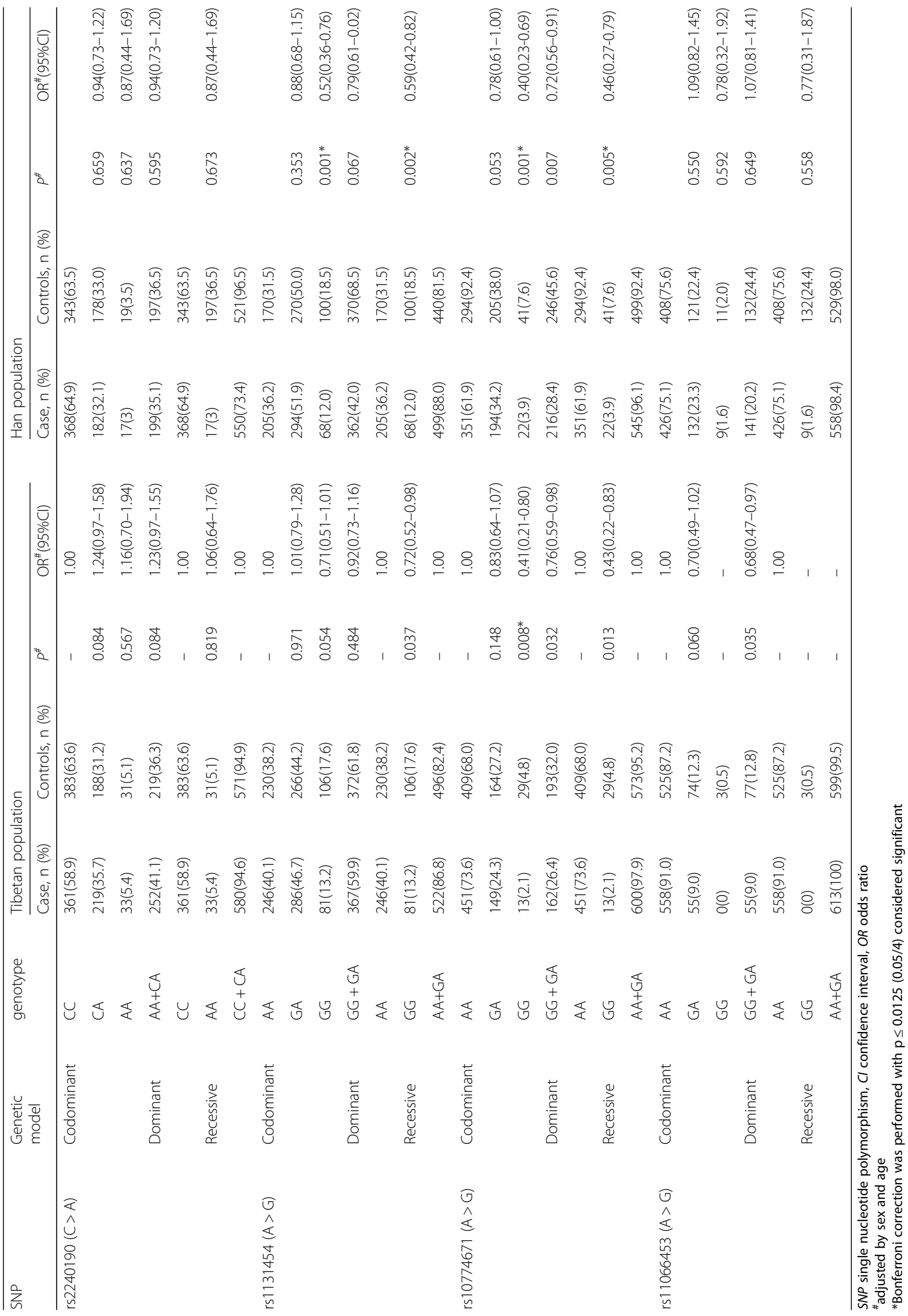


Table 4 Haplotype analyses in the Tibetan population

\begin{tabular}{lllllllll}
\hline \multicolumn{7}{l}{ Tibetan population } & \multicolumn{7}{l}{ Han population } \\
\hline Haplotype & Cases, $\mathrm{n}(\%)$ & Controls, $\mathrm{n}(\%)$ & $\mathrm{P}$ & $\mathrm{OR}$ & Cases, $\mathrm{n}(\%)$ & Controls, $\mathrm{n}(\%)$ & $\mathrm{P}$ & OR \\
AGAA & $198(16.1)$ & $162(13.5)$ & 0.038 & $1.27(1.01-1.59)$ & $132(11.4)$ & $113(10.4)$ & 0.454 & $1.11(0.85-1.45)$ \\
AGGA & $53(4.3)$ & $80(6.6)$ & 0.018 & $0.65(0.46-0.93)$ & $74(6.4)$ & $94(8.7)$ & 0.043 & $0.72(0.53-0.99)$ \\
CAAA & $690(56.3)$ & $640(53.2)$ & 0.035 & $1.19(1.01-1.40)$ & $538(46.4)$ & $459(42.5)$ & 0.052 & $1.18(1.00-1.40)$ \\
CAAG & $54(4.4)$ & $78(6.5)$ & 0.034 & $0.68(0.48-0.97)$ & $150(12.9)$ & $142(13.1)$ & 0.926 & $0.99(0.77-1.27)$ \\
CGAA & $75(6.1)$ & $94(7.8)$ & 0.153 & $0.80(0.58-1.09)$ & $60(5.2)$ & $69(6.4)$ & 0.227 & $0.80(0.56-1.15)$ \\
CGGA & $121(9.9)$ & $141(11.7)$ & 0.207 & $0.85(0.66-1.10)$ & $190(16.4)$ & $193(17.9)$ & 0.357 & $0.90(0.72-1.12)$ \\
Other $^{\mathrm{a}}$ & $35(2.9)$ & $9(0.7)$ & - & & $15(1.4)$ & $11(0.9)$ & & \\
\hline
\end{tabular}

$\mathrm{Cl}$ confidence interval, $\mathrm{OR}$ odds ratio

${ }^{\text {a }}$ Those lowest frequency threshold $(\mathrm{LFT})<0.03$ were pooled in this part

Bonferroni correction was performed with $p \leq 0.008(0.05 / 6)$ considered significant

For each haplotype, alleles are arranged in order of rs2240190, rs1131454, 10,774,671 and 11,066,453

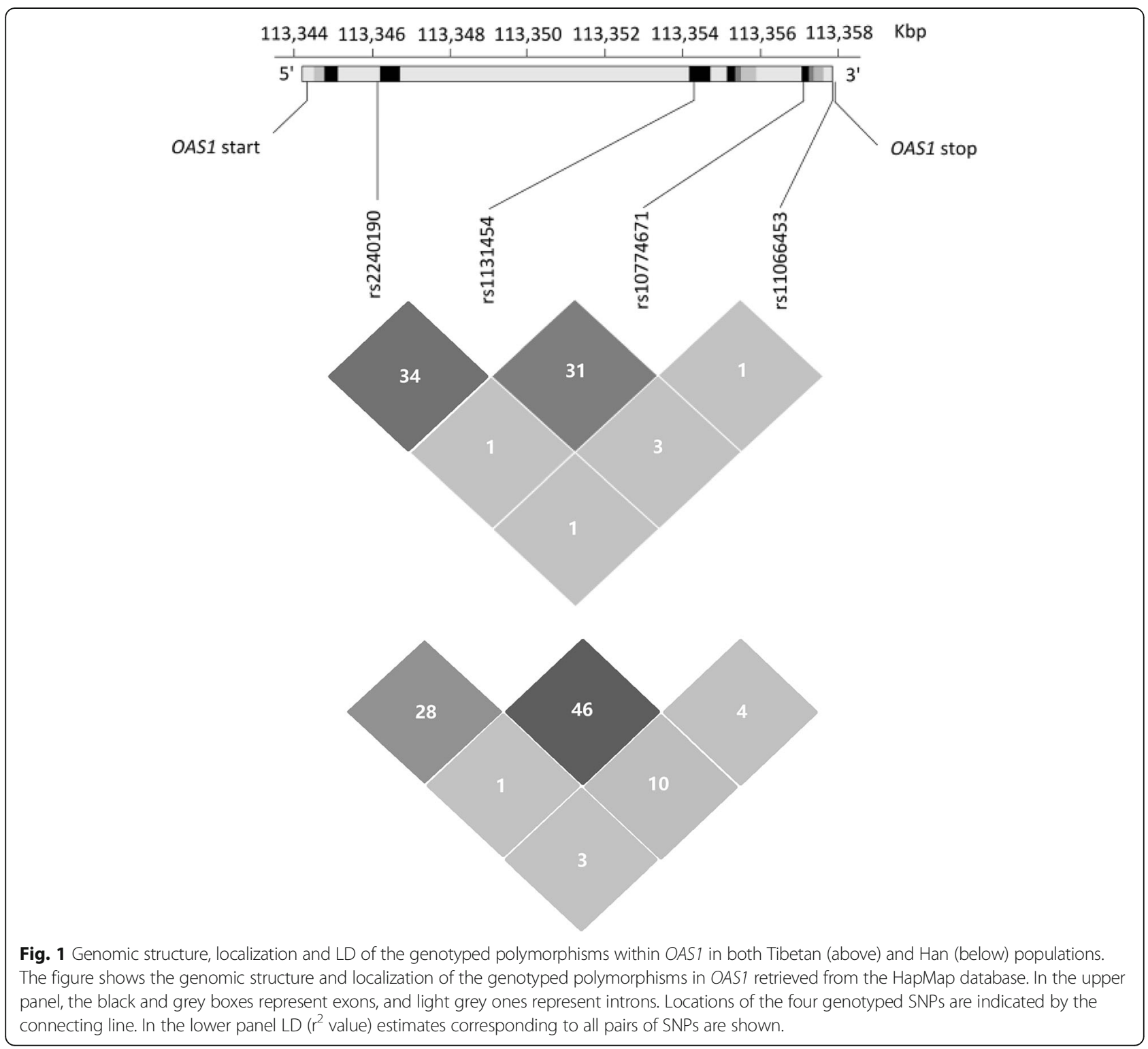




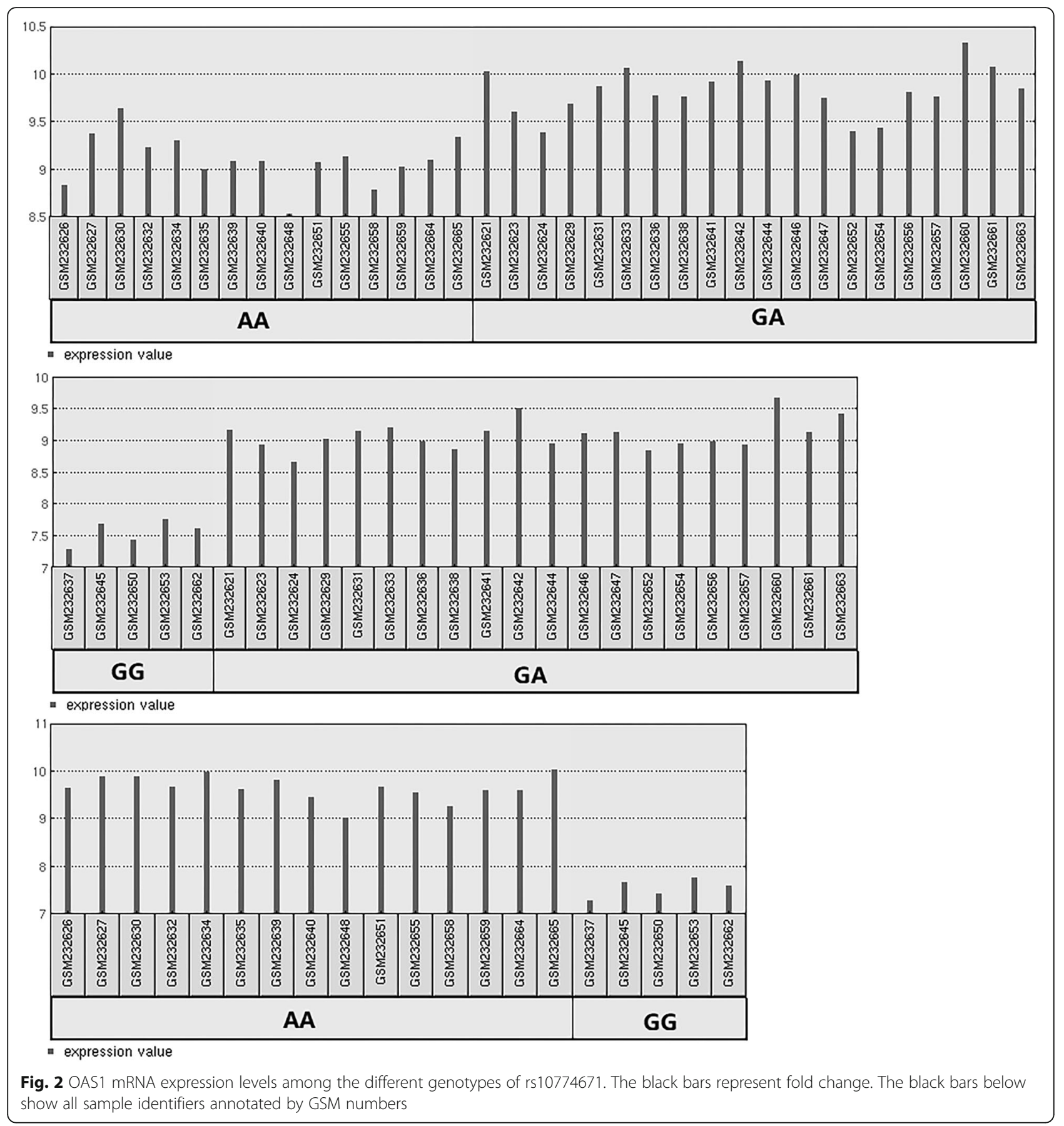

roles in other cellular functions. The role of OAS1 in immune-related diseases has been shown by a number of association studies $[25,26]$. It is well known that the immune response against TB plays a critical role in the progression of $M T B$ infection. Another explanation is that the OAS1 gene affects susceptibility to TB through the type II IFN pathway $[14,15]$. Type II IFN (IFN- $\gamma$ ) is critical for host defence against certain bacterial and parasitic pathogens [37]. IFN- $\gamma$ can activate infected host macrophages to inhibit the replication of MTB directly
[38]. In addition, OAS gene expression was regulated by both type I and type II IFNs [39]. A previous study suggested that IFN- $\gamma$ could increase the levels of OAS mRNA [40]. Furthermore, OAS1 gene up-regulation was observed in several gene expression signatures that differentiated active TB from latent TB infection $[41,42]$. It was also established that there was a prominent correlation between OAS expression and TB [14]. Moreover, data from Noguchi et al. revealed the potential role of OAS1 polymorphisms in respiratory infection [27]. 
Therefore, we assume that the polymorphism in the $O A S$ gene may be associated with the risk of TB infection through the type II IFN pathway.

As a functional $O A S 1$ polymorphic marker, rs10774671 was reported to be associated with disease/viral infection $[36,43]$. rs 10774671 is located at the last nucleotide of intron 6 in $O A S 1$, which acts as a splice-acceptor site for exon 7 . The $\mathrm{G}$ allele was demonstrated to allow splicing to occur leading to the production of a p46 form with high enzymatic OAS activity [20]. The rs10774671 A allele usually prevents splicing at this site and thus splicing happens further downstream, resulting in the p48 and p52 forms associated with low OAS enzymatic activity. rs10774671 could also control other splice variants of OAS including p42 and p44 [20]. In addition, the HapMap genotypic data (Fig. 2) of rs10774671 suggested that individuals with GG genotype had lower expression levels than those with AA and GA genotypes. However, rs10774671 GG genotype was reported to be associated with the highest enzyme activity of OAS1 in unstimulated lymphocytes [44]. The inconsistent results between mRNA expression and enzyme activity may attribute to the reason that gene expressions at the transcriptional and translational levels might be different. Our data were partly consistent with these observations, in that the G allele and GG genotype had significant protective effects against $T B$, whereas the AG genotype was not associated with TB. Furthermore, this result was validated in the Chinese Han population. Considering the aforementioned functional effects of rs10774671 on phenotypes, we speculate that rs10774671 could alter OAS1 expression levels precluding the type I IFN pathway resulting in a protective response.

In order to detect whether two or more SNPs interact either directly or indirectly to change TB risk separate from their independent effects, we conducted a gene-gene interaction analysis. We did not find any significant differences in single SNP analysis between cases and controls groups for rs2240190, rs1131454 and 11,066,453. However, rs2240190, rs1131454 and rs10774671 formed the best gene-gene interaction model. This result was consistent with the phenomenon that most diseases including TB are caused by multiple factors and gene-gene or gene-environment interactions are characteristic of the genetic factors. In addition, we also conducted a haplotype analysis which was based on the association between a polymorphism and the ancestral haplotype [45]. However, inconsistent with the gene-gene interaction result, there was no significant association between haplotypes and TB. Since the haplotype analysis approach was used for analyzing haplotype effects at numerous closely linked loci, and the gene-gene interaction analysis could be used for analyzing the unlinked loci $[46,47]$. We speculate the discrepant results are likely attributable to different methods. rs2240190 is located in intron 1 and it may have an alternative splicing regulatory effect, based on the Functional Single Nucleotide Polymorphism database [48]. To the best of our knowledge, no previous studies investigated the association between rs2240190 and disease. Only one study suggested that this genetic polymorphism was not in LD with rs10774671 [27]. rs1131454 (formerly rs3741981), in the evolutionarily conserved exon 3 , is nonsynonymous (the $\mathrm{G} \rightarrow \mathrm{A}$ transition results in a $\mathrm{G} \rightarrow \mathrm{S}$ amino acid change). This polymorphic locus is close to the dsRNA binding domain of all OAS1 isoforms. In general, the AA, GA and GG genotypes of rs1131454 lead to low, intermediate and high OAS1 enzyme activity, respectively [44]. This polymorphic locus was demonstrated to be functional in the progression of the severe acute respiratory syndrome [49]. In our study, we demonstrated that the G allele and GG genotype were associated with TB in the Han population.

Genome-wide association studies with longitudinal data found that rs11066453 was a significant disease susceptibility locus, as confirmed by the Health Examination cohort [50]. Furthermore, the rs11066453 polymorphism was strongly related to OAS1 mRNA expression levels in Epstein-Barr virus infection [51], and the monoallelically expressed OAS1 gene was also significantly associated with the gamma-glutamyltransferase level [28]. This strong association of the OAS1 gene that contains the rs11066453 polymorphism in the development and progression of human diseases may be due to it affecting OAS1 expression. However, inconsistent with these findings, there was no significant association with TB susceptibility detected in the present study.

There are several limitations in our study. First, lack of data regarding the association between other genes in the OAS cluster and TB limited our understanding of genetic mechanisms regulating the pathogenesis of TB. Second, our study lacked clinical information for the Tibetans, which limited the analysis of the association with clinical characteristics. Finally, functional validation of the included SNPs was not carried out. As a result, the true causal allele underlying the genetic association result is still unknown.

In conclusion, we were the first to investigate the association between OAS1 polymorphisms and TB in the Chinese Tibetan population and validated the results in an independent cohort. We found that rs10774671, located in the OAS1 gene region is associated with susceptibility to TB in Tibetans and may act as a protective factor against $\mathrm{TB}$, which was validated in the Chinese Han population. In addition, we also found that rs1131454 was a protective factor against $\mathrm{TB}$ in the Chinese Han population. rs2240190, rs1131454 and rs10774671 formed the best gene-gene interaction model in MDR analysis and may be considered as one of the 
multiple contributors to the progress of this complicated disease. However, in order to get more detailed mechanistic insight into the pathway of how OAS1 may act on TB, there is a need for further research in this field. Similar studies in different populations, gene-environment interactions analysis and functional studies are warranted to confirm and reinforce our results.

\section{Abbreviations}

Cl: Confidence interval; HIV: Human immunodeficiency virus; HWE: HardyWeinberg equilibrium; IFN: Interferon; iMLDR: Improved multiple ligase detection reaction; LD: Pairwise linkage disequilibrium; MAF: Minor allele frequency; MDR: Multifactor dimensionality reduction; MTB: Mycobacterium tuberculosis; OAS1: 2'-5'-oligoadenylate synthetase 1; OR: Odds ratio; RNase L: Ribonuclease L; RR: Relative risks; SNPs: Single-nucleotide polymorphisms; TB: Tuberculosis

\section{Acknowledgements}

The authors are grateful for all the cases and the controls who volunteered for participating in the study.

\section{Funding}

This work was supported in part by the National Natural Science Foundation of China (Beijing, China) Grants 81870015, 81072432. This funding supported the costs of the design of the study and collection, analysis, and interpretation of data and in writing the manuscript.

\section{Availability of data and materials}

The data analyzed during the study are not publicly available as is they are to be used for further research work on the topic. However, the data can be obtained from the authors upon request.

\section{Authors' contributions}

All authors read and approved the final manuscript. Conceived and designed the experiments: JQH. Performed the experiments: YW SQW GC. Analyzed the data: SQW JQH. Contributed reagents/materials/analysis tools: MMZ MGW. Wrote the paper: SQW JQH. Obtained ethical permission for the use of blood when taking samples: YW SQW JQH.

\section{Ethics approval and consent to participate}

The study was approved by the ethical committee of the West China Hospital of Sichuan University (the NO.131 file). The ethical approval is as follows. All participants in both groups voluntarily joined this study with written informed consents. A sample of $5 \mathrm{ml}$ of peripheral blood was obtained from each participant.

All procedures performed in studies involving human participants were in accordance with the ethical standards of the institutional and/or national research committee and with the 1964 Helsinki declaration and its later amendments or comparable ethical standards.

\section{Consent for publication}

Not applicable.

\section{Competing interests}

The authors declare that they have no conflict of interest.

\section{Publisher's Note}

Springer Nature remains neutral with regard to jurisdictional claims in published maps and institutional affiliations.

\section{Author details}

'Department of Respiratory and Critical Care Medicine, West China Hospital, Sichuan University, No. 37, Guo Xue Alley, Chengdu 610041, Sichuan, People's Republic of China. 'Division of Geriatrics, Sichuan Provincial People's Hospital, Chengdu, Sichuan, China.
Received: 14 March 2018 Accepted: 19 November 2018

Published online: 29 November 2018

\section{References}

1. World Health Organization (2018). Global tuberculosis report 2018. http:// www.who.int/tb/publications/global_report/en/(accessed Sep 26, 2018).

2. Comstock GW, et al. The prognosis of a positive tuberculin reaction in childhood and adolescence. Am J Epidemiol. 1974;99(2):131-8.

3. van de Vosse $E$, et al. Human genetics of intracellular infectious diseases: molecular and cellular immunity against mycobacteria and salmonellae. Lancet Infect Dis. 2004;4(12):739-49.

4. Newport MJ, et al. A mutation in the interferon-gamma-receptor gene and susceptibility to mycobacterial infection. N Engl J Med. 1996:335(26):1941-9.

5. Manca C, et al. Virulence of a Mycobacterium tuberculosis clinical isolate in mice is determined by failure to induce Th1 type immunity and is associated with induction of IFN-alpha /beta. Proc Natl Acad Sci U S A. 2001;98(10):5752-7.

6. de Paus RA, et al. Inhibition of the type I immune responses of human monocytes by IFN-alpha and IFN-beta. Cytokine. 2013;61(2):645-55.

7. van de Vosse $\mathrm{E}$, et al. Genetic deficiencies of innate immune signalling in human infectious disease. Lancet Infect Dis. 2009;9(11):688-98.

8. Flynn $\mathrm{J} L$, et al. An essential role for interferon gamma in resistance to Mycobacterium tuberculosis infection. J Exp Med. 1993;178(6):2249-54.

9. Hovnanian A, et al. The human 2',5'-oligoadenylate synthetase locus is composed of three distinct genes clustered on chromosome 12q24.2 encoding the 100-, 69-, and 40-kDa forms. Genomics. 1998;52(3):267-77.

10. Sadler AJ, Williams BR. Interferon-inducible antiviral effectors. Nat Rev Immunol. 2008:8(7):559-68.

11. Hovanessian AG, Justesen J. The human 2'-5'oligoadenylate synthetase family: unique interferon-inducible enzymes catalyzing $2^{\prime}-5^{\prime}$ instead of $3^{\prime}-5$ phosphodiester bond formation. Biochimie. 2007;89(6-7):779-88.

12. Domingo-Gil E, Esteban M. Role of mitochondria in apoptosis induced by the 2-5A system and mechanisms involved. Apoptosis. 2006;11(5):725-38.

13. Yaffe $\mathrm{A}$, et al. Inhibition of 2-5A synthetase expression by antisense RNA interferes with interferon-mediated antiviral and antiproliferative effects and induces anchorage-independent cell growth. Cell Growth Differ. 1996;7(8):969-78.

14. Leisching $\mathrm{G}$, et al. OAS1, 2, and 3: significance during active tuberculosis? J Infect Dis. 2018;217(10):1517-21.

15. Leisching $\mathrm{G}$, et al. The association of OASL and type I Interferons in the pathogenesis and survival of intracellular replicating bacterial species. Front Cell Infect Microbiol. 2017;7:196.

16. Justesen J, et al. Gene structure and function of the 2'-5'-oligoadenylate synthetase family. Cell Mol Life Sci. 2000;57(11):1593-612.

17. Ibsen MS, et al. The 2'-5'-oligoadenylate synthetase 3 enzyme potently synthesizes the 2'-5'-oligoadenylates required for RNase $L$ activation. J Virol. 2014;88(24):14222-31.

18. Li $Y$, et al. Activation of RNase $L$ is dependent on OAS3 expression during infection with diverse human viruses. Proc Natl Acad Sci U S A. 2016:113(8):2241-6.

19. Dunn GP, et al. The immunobiology of cancer immunosurveillance and immunoediting. Immunity. 2004;21(2):137-48.

20. Lim JK, et al. Genetic variation in OAS1 is a risk factor for initial infection with West Nile virus in man. PLoS Pathog. 2009;5(2):e1000321.

21. Zhao Y, et al. Evaluate the relationship between polymorphisms of OAS1 gene and susceptibility to chronic hepatitis $C$ with high resolution melting analysis. Clin Exp Med. 2013;13(3):171-6.

22. Chaaithanya IK, et al. Association of Oligoadenylate Synthetase Gene Cluster and DC-SIGN (CD209) gene polymorphisms with clinical symptoms in Chikungunya virus infection. DNA Cell Biol. 2016;35(1):44-50.

23. Thamizhmani $R$, Vijayachari P. Association of dengue virus infection susceptibility with polymorphisms of 2'-5'-oligoadenylate synthetase genes: a case-control study. Braz J Infect Dis. 2014;18(5):548-50.

24. Haralambieva $\mathbb{I H}$, et al. Genetic polymorphisms in host antiviral genes: associations with humoral and cellular immunity to measles vaccine. Vaccine. 2011;29(48):8988-97.

25. Tessier MC, et al. Type 1 diabetes and the OAS gene cluster: association with splicing polymorphism or haplotype? J Med Genet. 2006;43(2):129-32.

26. Fedetz M, et al. OAS1 gene haplotype confers susceptibility to multiple sclerosis. Tissue Antigens. 2006;68(5):446-9. 
27. Noguchi S, et al. Differential effects of a common splice site polymorphism on the generation of OAS1 variants in human bronchial epithelial cells. Hum Immunol. 2013;74(3):395-401.

28. Park YM, et al. Genome-wide detection of allelic gene expression in hepatocellular carcinoma cells using a human exome SNP chip. Gene. 2014; 551(2):236-42.

29. Hall NB, et al. Polymorphisms in TICAM2 and IL1B are associated with TB. Genes Immun. 2015;16(2):127-33.

30. Sabri A, et al. Association study of genes controlling IL-12-dependent IFN-gamma immunity: STAT4 alleles increase risk of pulmonary tuberculosis in Morocco. J Infect Dis. 2014;210(4):611-8.

31. Chen $\mathrm{C}$, et al. A rare variant at $11 \mathrm{p} 13$ is associated with tuberculosis susceptibility in the Han Chinese population. Sci Rep. 2016;6:24016.

32. He S, et al. Association of IL4, IL6, and IL10 polymorphisms with pulmonary tuberculosis in a Tibetan Chinese population. Oncotarget. 2018;9(23):16418-26.

33. Wang $L$, et al. The fifth national tuberculosis epidemiological survey in 2010 . Chin J Antituber. 2012;34(8):485-508.

34. Schneider WM, et al. Interferon-stimulated genes: a complex web of host defenses. Annu Rev Immunol. 2014;32:513-45.

35. Tsutsui-Takeuchi $\mathrm{M}$, et al. Roles of retinoic acid-inducible gene--llike receptors (RLRs), toll-like receptor (TLR) 3 and 2'-5' oligoadenylate synthetase as viral recognition receptors on human mast cells in response to viral infection. Immunol Res. 2015;61(3):240-9.

36. Liu X, et al. A functional variant in the OAS1 gene is associated with Sjogren's syndrome complicated with HBV infection. Sci Rep. 2017; 7(1):17571.

37. Boehm U, et al. Cellular responses to interferon-gamma. Annu Rev Immunol. 1997;15:749-95.

38. MacMicking JD, et al. Immune control of tuberculosis by IFN-gammainducible LRG-47. Science. 2003;302(5645):654-9.

39. Zhou A, et al. Interferon action and apoptosis are defective in mice devoid of 2',5'-oligoadenylate-dependent RNase L. EMBO J. 1997;16(21):6355-63.

40. Corrias MV, et al. Induction of $2.5 \mathrm{OAS}$ gene expression and activity is not sufficient for IFN-gamma-induced neuroblastoma cell differentiation. Int J Cancer. 1995;62(2):223-9.

41. Berry MP, et al. An interferon-inducible neutrophil-driven blood transcriptional signature in human tuberculosis. Nature. 2010; 466(7309):973-7.

42. Ottenhoff $\mathrm{TH}$, et al. Genome-wide expression profiling identifies type 1 interferon response pathways in active tuberculosis. PLoS One. 2012; 7(9):e45839.

43. Bader El Din NG, et al. Impact of OAS1 exon 7 rs10774671 genetic variation on liver fibrosis progression in Egyptian HCV genotype 4 patients. Viral Immunol. 2015;28(9):509-16.

44. Bonnevie-Nielsen $\mathrm{V}$, et al. Variation in antiviral 2',5'-oligoadenylate synthetase ( $\left(2^{\prime} 5^{\prime} \mathrm{AS}\right)$ enzyme activity is controlled by a single-nucleotide polymorphism at a splice-acceptor site in the OAS1 gene. Am J Hum Genet. 2005; $76(4): 623-33$

45. Gabriel SB, et al. The structure of haplotype blocks in the human genome. Science. 2002;296(5576):2225-9.

46. Thornton-Wells TA, et al. Genetics, statistics and human disease: analytical retooling for complexity. Trends Genet. 2004;20(12):640-7.

47. Cordell HJ, et al. Case/pseudocontrol analysis in genetic association studies: a unified framework for detection of genotype and haplotype associations, gene-gene and gene-environment interactions, and parent-of-origin effects. Genet Epidemiol. 2004;26(3):167-85.

48. Lee PH, Shatkay H. F-SNP: computationally predicted functional SNPs for disease association studies. Nucleic Acids Res. 2008:36(Database issue):D820-4.

49. Hamano $E$, et al. Polymorphisms of interferon-inducible genes OAS-1 and MXA associated with SARS in the Vietnamese population. Biochem Biophys Res Commun. 2005;329(4):1234-9.

50. Lee $\mathrm{Y}$, et al. On the analysis of a repeated measure design in genome-wide association analysis. Int J Environ Res Public Health. 2014;11(12):12283-303.

51. Kim YJ, et al. Large-scale genome-wide association studies in east Asians identify new genetic loci influencing metabolic traits. Nat Genet. 2011; 43(10):990-5.

\section{Ready to submit your research? Choose BMC and benefit from:}

- fast, convenient online submission

- thorough peer review by experienced researchers in your field

- rapid publication on acceptance

- support for research data, including large and complex data types

- gold Open Access which fosters wider collaboration and increased citations

- maximum visibility for your research: over $100 \mathrm{M}$ website views per year

At BMC, research is always in progress.

Learn more biomedcentral.com/submissions 